\title{
Encoded Simultaneous Sources Full-Waveform Inversion based on the phase rotation of wavefield in the time domain
}

\author{
Edwin Fagua Duarte*, Carlos A. N. da Costa, Gilberto Corso and Joao M. de Araujo \\ DFTE - CISCPET /Universidade Federal do Rio Grande do Norte
}

Copyright 2019, SBGf - Sociedade Brasileira de Geofísica.

This paper was prepared for presentation at the $16^{t h}$ International Congress of the Brazilian Geophysical Society, held in Rio de Janeiro, Brazil, 19-22 August, 2019.

Contents of this paper were reviewed by the Technical Committee of the $16^{\text {th }}$ International Congress of the Brazilian Geophysical Society and do not necessarily represent any position of the SBGf, its officers or members. Electronic reproduction or storage of any part of this paper for commercial purposes without the written consent of the Brazilian Geophysical Society is prohibited.

\begin{abstract}
Encoding techniques are powerful strategies to speedup seismic inversion processes based on full-waveform inversion. In this work, we introduce a new simultaneous sources encoding strategy for time domain full-waveform inversion based on the phase rotation wavefield in the time domain constructed with help of the Hilbert transform. This encoding strategy consists in applying a random phase rotation to each shot gather. In addition, we combine our encoding scheme with restarted L-BFGS scheme. We have tested our scheme using synthetic data set from 2D SEG/EAGE overthrust model. In our tests, we have compared the effectiveness of our scheme with the conventional polarity encoding strategy. In this comparison, our scheme has shown to be more effective to mitigate the crosstalk noise than the polarity encoding strategy. Furthermore, our scheme showed an improvement of the convergence speed in comparison with polarity encoding.
\end{abstract}

\section{Introduction}

In principle, seismic inversion processes based on fullwaveform seismic inversion (FWI) can recover quantitative medium properties of the subsurface with high-resolution (Lailly, 1983; Tarantola, 1984; Virieux and Operto, 2009). However, these inversion processes are computationally expensive (Krebs et al., 2009; Ben-Hadj-Ali et al., 2011; Haffinger et al., 2013; da Costa et al., 2018), which limits its application to realistic seismic inversion problems from exploration seismology. In a standard gradient based FWI, where the gradient of objective function is computed via adjoint-state method (Plessix, 2006), the computational cost, in term of time spent, is directly related to the number of available sources (especially in the time domain), because during the inversion process is required to perform at least two modelling per iteration for each source.

In order to improve the computational speed of the gradient based FWI, Krebs et al. (2009) proposed the encoded simultaneous-source FWI, where the gradient of the objective function is calculated for all shots simultaneously. Indeed, this is a powerful strategy to speed-up the FWI, because in this formulation the number of modelling required to construct the gradient of the objective function is reduced to two per iteration. However, a drawback of this scheme is the crosstalk noise that arises from the correlation of the forward- and backward- wavefields from different shots. A way to attenuate these noise is applying to each shot an operation called encoding (Krebs et al., 2009). The encoding in the time domain, in general, is formulated as a randomly polarity scheme (so-called polarity encoding technique), where each shot is weighted by a polarity number +1 or -1 chosen randomly (Krebs et al., 2009; Rao and Wang, 2017). In the frequency domain, the encoding is formulated as a phaseshift scheme (so-called phase encoding technique), where a phase-shift, with a random or deterministic choice of phases, is applied to each shot (Romero et al., 2000; Jing et al., 2000; Ben-Hadj-Ali et al., 2009; Ben-Hadj-Ali et al., 2011). For frequency domain FWI, the random phase encoding has been demonstrated to be more effective than the deterministic phase encoding (Ben-Hadj-Ali et al., 2009).

In the same way as the frequency domain, in the time domain a phase rotation can be applied using the concepts of analytic signal and Hilbert transform. Thus, we proposed an alternative encoding strategy where each shot is encoded by applying a phase rotation in the calculation of gradient of the cost function. The phase angle for each shot is chosen randomly. We combined this codification strategy with the restarted L-BFGS method for quasiNewton inversion showed in Rao and Wang (2017) and Rao et al. (2019).

\section{Methodology}

\section{Phase Rotation}

The phase rotation for a time series can be calculated using the concept of analytic signal, that is widely used in seismic processing and interpretation to obtain mathematical attributes of seismic traces as instantaneous phase and the signal envelope (Taner et al., 1979; Wang, 2007; Barnes, 2007). According to Bracewell (1999); Taner et al. (1979) a real times series $x(t)$ can be interpreted as the real part of a complex function called the analytic signal $F(t)$ defined as

$$
F(t)=x(t)+\imath \operatorname{Hilb}\{x(t)\}
$$

Where Hilb \{\} is the Hilbert transform of $x(t)$, defined as

$$
\operatorname{Hilb}\{x(t)\}=P . V \cdot \frac{1}{\pi} \int_{-\infty}^{\infty} \frac{x\left(t^{\prime}\right)}{t^{\prime}-t} d t^{\prime}
$$

where P.V. is the Cauchy principal value of the convolutional integral. Numerically is more convenient to calculate the hilbert transform using the definition in terms of Fourier 
transform

$$
\operatorname{Hilb}(x(t))=\mathscr{F}^{-1}\{-\imath \operatorname{Sign}(\omega) \mathscr{F}\{x(t)\}\}
$$

where $\omega$ is the angular frequency, $\mathscr{F}$ is the Fourier transform operator and $\operatorname{Sign}()$ is the signal function. The Hilbert transform is commonly interpreted as a $90^{\circ}$ phase rotation of the original time series. At the same time, the original time series is referred as the $0^{\circ}$ version. Hence, using the real and imaginary parts of analytic signal $F(t)$, a $\varphi$ degrees phase rotation $x_{r o t}(t ; \varphi)$ of the time series $x(t)$ is calculated as (See equation A-1 from Barnes, 2007)

$$
x_{\text {rot }}(t ; \varphi)=x(t) \cos (\varphi)+\operatorname{Hilb}\{x(t)\} \sin (\varphi)
$$

\section{Encoding Strategies Tests}

In order to show the performance of the phase rotation encoding we tested two encoding strategies. In the first one, we applied a phase rotation to each shot. The shot traces were rotated using the equation (4) choosing the angle $\varphi$ between 0 and $\pi$. All the traces of a shot were rotated with the same angle but the angle is different for all shots and was chosen randomly. The phase rotation using the angles 0 and $\pi$ is equivalent to the two values +1 and -1 of the polarity encoding strategy. This strategy aimed to sweep all the phase angles between the values of the polarity strategy. The second strategy tested is the polarity encoding mentioned above (Krebs et al., 2009; Rao and Wang, 2017).

As mentioned in the introduction, our encoding strategy is combined with the restarted L-BFGS method described in Rao and Wang (2017). This strategy uses the conventional L-BFGS quasi-Newton method (Nocedal and Wright, 2006), applying an initial encoding for the shots and keeping this codification for $m$ iterations. After this, the codification is changed until reaching $l$ iterations, where the L-BFGS is restarted, that is, keeping just the last gradient to avoid the initial linear search. This procedure was repeated until reach convergence. According to Rao and Wang (2017), this strategy increases the randomness of encoding and, therefore attenuates the crosstalk.

For the two strategies, phase rotation and polarity encoding the cost function $\Phi$ was calculated as

$$
\Phi=\frac{1}{2}\left\|D_{\text {sim }}-\sum_{i=1}^{N_{s}} d_{o b s}(i)\right\|_{2}^{2}
$$

where $d_{o b s}(i)$ is the observed data for shot $i$ and $N_{s}$ is the number of sources. All the observed shots must be stacked to be compared with the simulated data. $D_{\text {sim }}$ is the simulated super-shot were all the shots were propagated at the same time, i.e, is the solution at the receiver positions of the equation

$$
\nabla^{2} D_{s i m}-\frac{1}{c^{2}(\vec{x})} \frac{\partial^{2} D_{s i m}}{\partial t^{2}}=\sum_{i=1}^{N_{s}} s(i, t)
$$

where $s(i, t)$ is the source wavelet at the position of shot $i$. The cost function does not depend on the encoding strategy, which implies that the evolution of cost function is comparable for the different encoding strategies.

\section{Encoded Gradient Calculation}

In the conventional FWI, the gradient of cost function using $N_{s}$ sources is calculated as (Plessix, 2006)

$$
\nabla \Phi=-\sum_{i=1}^{N_{s}} \int_{0}^{T} R_{i}(\vec{x}) \frac{\partial^{2} P_{i}(\vec{x})}{\partial t^{2}} d t
$$

Where $P_{i}$ is the forward propagated wavefield of a synthetic wavelet at shot position $i, R_{i}$ is the back propagated wavefield where the source term is the difference between simulated and original shots and $T$ is the record length. Figure 1(e) shows an example of conventional gradient calculated with equation (7) for the model of Figure 2(b). For encoded simultaneous shots FWI, the gradient is calculated as

$$
\nabla \Phi=-\int_{0}^{T} R_{\text {sim }}(\vec{x}) \frac{\partial^{2} P_{\text {sim }}(\vec{x})}{\partial t^{2}} d t
$$

where $P_{\text {sim }}$ is calculated in the same way as equation (6) but the source term is encoded, that means, $P_{\text {sim }}$ is solution of equation

$$
\nabla^{2} P_{s i m}-\frac{1}{c^{2}(\vec{x})} \frac{\partial^{2} P_{s i m}}{\partial t^{2}}=\sum_{i=1}^{N_{s}} e_{i} \otimes s(i, t)
$$

The notation in equation (9) is similar to the used in Krebs et al. (2009) but the operation $e_{i} \otimes$ is not a convolution but is any operation on the time series, in this case, a phase rotation. The backpropagated wavefield is calculated solving the equation

$$
\nabla^{2} R_{\text {sim }}-\frac{1}{c^{2}(\vec{x})} \frac{\partial^{2} R_{\text {sim }}}{\partial t^{2}}=P_{\text {sim }}(-t)-\sum_{i=1}^{N_{s}} e_{i}^{*} \otimes d_{o b s}(i,-t)
$$

the $-t$ in the argument on the source term means that the wavefield is back-propagated. The $*$ on encoding operator means that this operation is back-propagated also. Using the fact that wave equation is linear, the equation (8) can be expressed as

$$
\begin{aligned}
\nabla \Phi= & -\sum_{i=1}^{N_{s}} \int_{0}^{T} e_{i}^{*} \otimes R_{i}(\vec{x}) \frac{\partial^{2} e_{i} \otimes P_{i}(\vec{x})}{\partial t^{2}} d t \\
& -\sum_{i \neq j}^{N_{s}} \int_{0}^{T} e_{i}^{*} \otimes R_{i}(\vec{x}) \frac{\partial^{2} e_{j} \otimes P_{j}(\vec{x})}{\partial t^{2}} d t
\end{aligned}
$$

The first term are the correlation of the forward and backpropagated wavefields coming from the same source, whereas the second term is the crosscorrelation of forward and backpropagated wavefields coming from different sources, i.e., the crosstalk noise. For any encoding strategy works, the first term of the equation (11), must recover the conventional gradient, that means, it must be equal to equation (7). For the polarity encoding is easy to notice that the first term of equation (11) recovers the equation (7), nevertheless, for phase rotation encoding this is not straightforward.

To show that phase rotation also recovers the conventional gradient, we calculated numerically the gradient for one 
shot using the equation (8) applying a $0^{\circ}$ phase rotation and a $53^{\circ}$ phase rotation that are showed in Figures 1(a) and 1 (b) respectively. For one shot the crosstalk term does not exist and, therefore, the gradient calculated by equation 8 is equivalent to the conventional gradient for this case. The result is the same for the two phase angle tested and, therefore, the first term of equation (11) reproduces the conventional gradient when phase rotation is used. The $53^{\circ}$ phase was chosen only as example, but the result is the same for any angle. One possible explanation for this is, that in the first term of equation (11), the rotation done on the forward wavefield is compensated by the rotation of the backpropagated wavefield.

Figures 1(c) and 1(d) showed the simultaneous encoded gradients for polarity encoding and phase rotation encoding respectively. It is noted that the gradient for phase rotation encoding has less crosstalk noise and shows more structures than the gradient corresponding to polarity encoding. An alternative way to show that the first term of (11) recovers the conventional gradient for polarity encoding is substracting the gradients of Figures $1(d)$ and $1(\mathrm{e})$. The result of this substraction is showed in Figure 1(f). We noted that the result of substraction only has crosstalk noise because it does not show any structure present on the conventional gradient.

\section{Numerical Experiments}

To illustrate the efficiency of the proposed methodology we used a synthetic dataset model, a $2 \mathrm{D}$ version of the SEG/EAGE Overthrust model, Figure 2(a). This model has 401 cells and 98 cells along the horizontal and vertical directions. The distance between cells was $h=50 \mathrm{~m}$. The observed and simulated data was calculated using the acoustic wave equation, employing a finite difference with a fourth order Laplacian and second order in time derivative together with a CPML absorbing boundary layer (Komatitsch and Martin, 2007; Pasalic and McGarry, 2010). The absorbing layer was applied at all sides of the model using a 30 cells width.

The source signature was a Ricker wavelet with a peak frequency of $f_{0}=6 \mathrm{~Hz}$. We employed 201 shots at the surface with 1500 samples and the time sample interval $0.004 \mathrm{~s}$. The receivers were positioned at the surface, one for each model column. The initial model (Figure 2(b)) was a smoothed version of the real model. To smooth the velocity model we calculated the average value of the $31 \times 21$ submatrix centered at each cell. We simulated a water layer in the first five rows of the model. These five rows were copied to the initial model, and the gradient was zeroed for these rows. This was done to avoid high amplitudes of the gradient close to the source positions. The parameters for restarted L-BFGS were $m=2$ and $l=5$ for all the numerical experiments.

The inverted models after 75 iterations are shown in Figures 2(c) and 2(d) for polarity and phase rotation encoding respectively. We notice that, at this intermediate phase of the inversion, the crosstalk noise is more mitigated using the rotation phase than in the polarity encoding. The final inverted models after 200 iterations are shown in figures $2(\mathrm{e})$ and 2(f) for polarity and phase rotation encoding respectively. We achieved an improvement in the definition of some events at the sides of the model, and the deepest part of the model below
$4 \mathrm{Km}$ depth. Figure 3 shows the cost function evolution versus the iterations for the two encoding strategies. The phase rotation encoding showed a faster convergence when compared with the polarity encoding.

Our results show that phase rotation encoding achieved similar inversion performance than polarity encoding but using only half of the iterations. Line searches were not carried out between iterations because, as explained in Nocedal and Wright (2006), the secant equation for the L-BFGS method scales the gradient in such a way that the step-length could be set up as 1 for all iterations. This is the reason for the local peaks in the cost function plot. One drawback of our method is the following: to perform the phase rotation it is necessary to calculate $2 N_{s}\left(N_{r}+1\right)$ Fourier transforms per iteration $\left(N_{r}\right.$ is the number of receivers). In our numerical experiments, the computational cost per iteration of phase rotation encoding was approximately 1.5 times the cost of gradient for polarity encoding. Nevertheless, the speed of convergence of phase rotation encoding compensated this extra time.

\section{Conclusions}

We proposed an alternative encoding strategy for calculation of gradient in the FWI time domain using simultaneous shots by applying a phase rotation to each shot of the survey. Our encoding methodology together to the restarted L-BFGS technique showed an improvement of the inversion when compared with the standard polarity strategy. The cost function of encoding strategy decreases faster than the standard encoding and the crosstalk noise attenuation has improved at the deepest part of the model. The behaviour of cost function shows that, with phase rotation encoding, we obtained a better performance than polarity encoding using fewer iterations.

\section{Acknowledgements}

The authors acknowledge the support of Conselho Nacional de Desenvolvimento Científico e Tecnológico, $\mathrm{CNPq}$ - Brazil. The authors also gratefully acknowledge the support from Shell Brasil through the "New Methods for Full Waveform Inversion" project at Universidade Federal do Rio Grande do Norte and the strategic importance of the support given by ANP through the R\&D levy regulation.

\section{References}

Barnes, A. E., 2007, A tutorial on complex seismic trace analysis: Geophysics, 72, W33-W43.

Ben-Hadj-Ali, H., S. Operto, and J. Virieux, 2009, Efficient 3D frequency-domain full waveform inversion (FWI) with phase encoding: 79th EAGE meeting, Amsterdam, The Netherlands, Expanded Abstracts, P004.

$\ldots, 2011$, An efficient frequency-domain full waveform inversion method using simultaneous encoded sources: Geophysics, 76, R109-R124.

Bracewell, R. N., 1999, The fourier transform and its applications, third ed.: McGraw-Hill Science/Engineering/Math.

da Costa, C. A., J. C. Costa, W. E. Medeiros, D. Verschuur, and A. K. Soni, 2018, Target-level waveform inversion: a prospective application of the convolution-type representation for the acoustic wavefield: Geophysical Prospecting, 67, 69-84.

Haffinger, P., A. Gisolf, and P. M. van den Berg, 


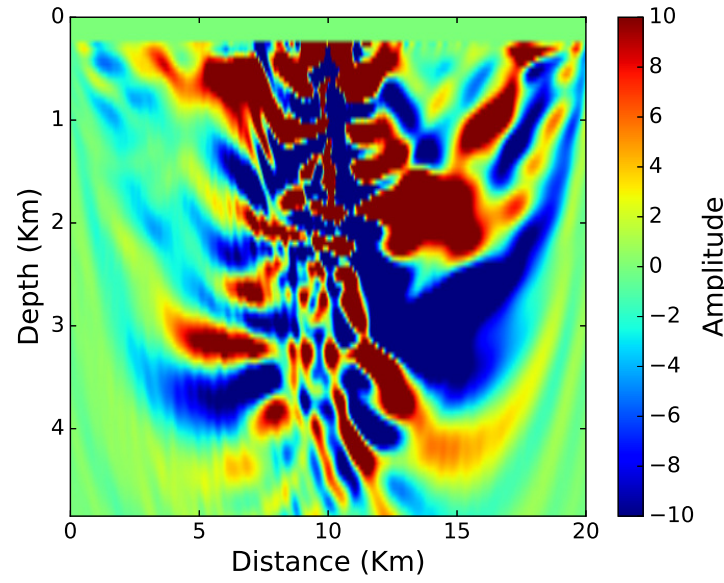

(a)

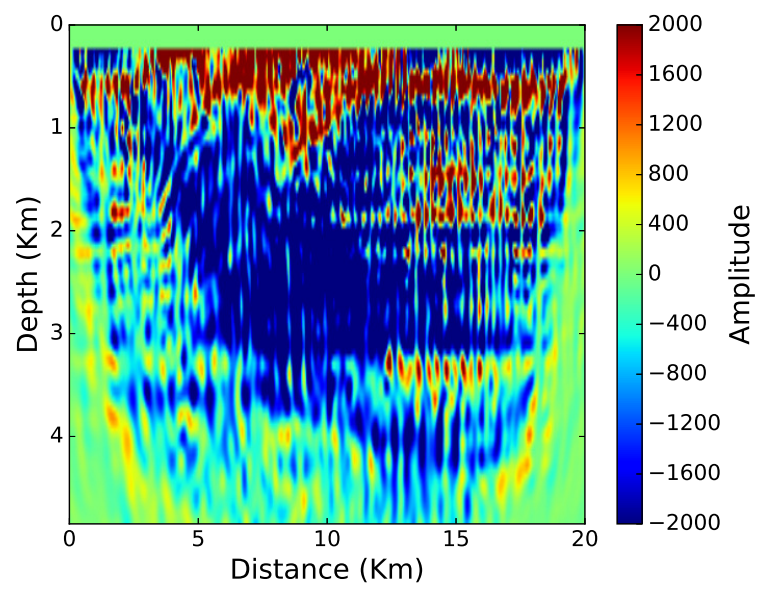

(c)

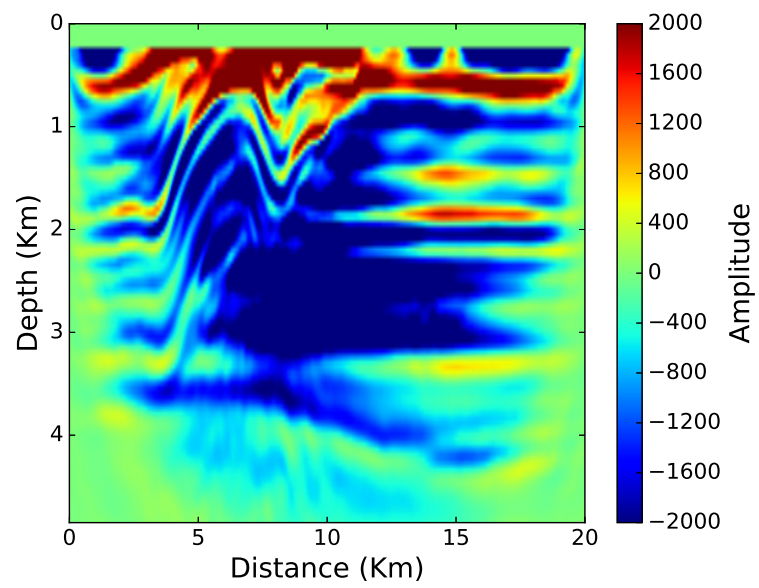

(e)

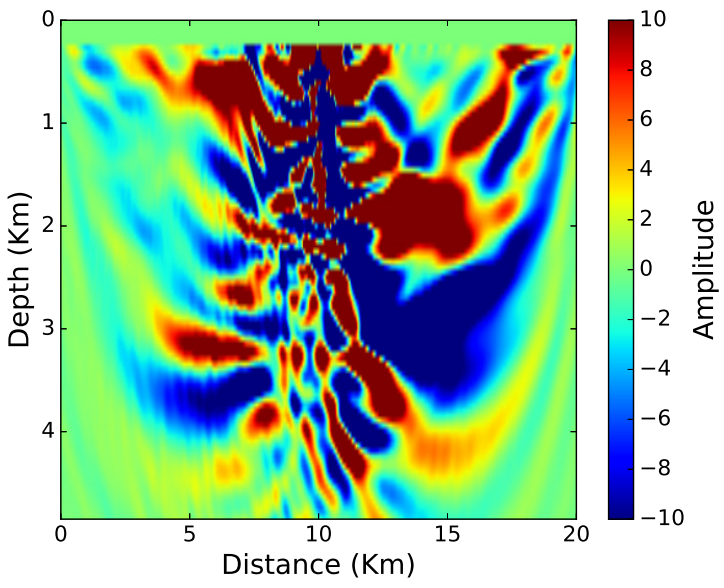

(b)

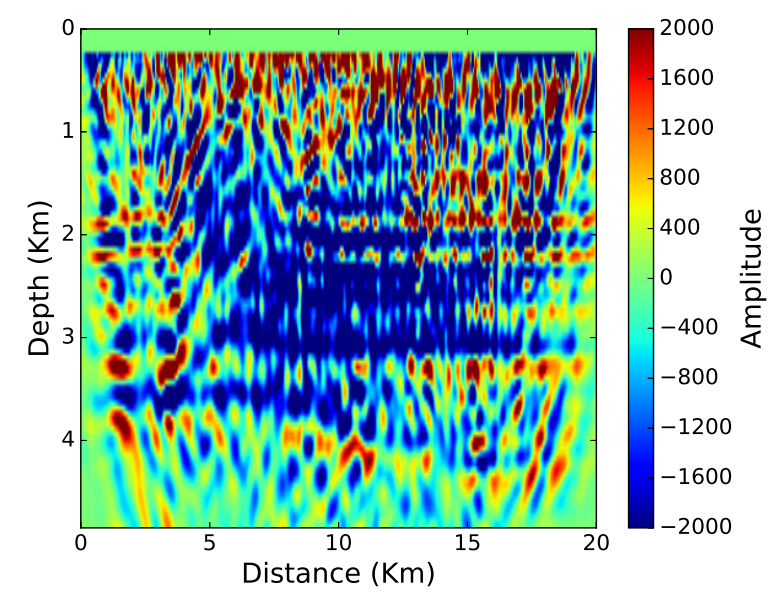

(d)

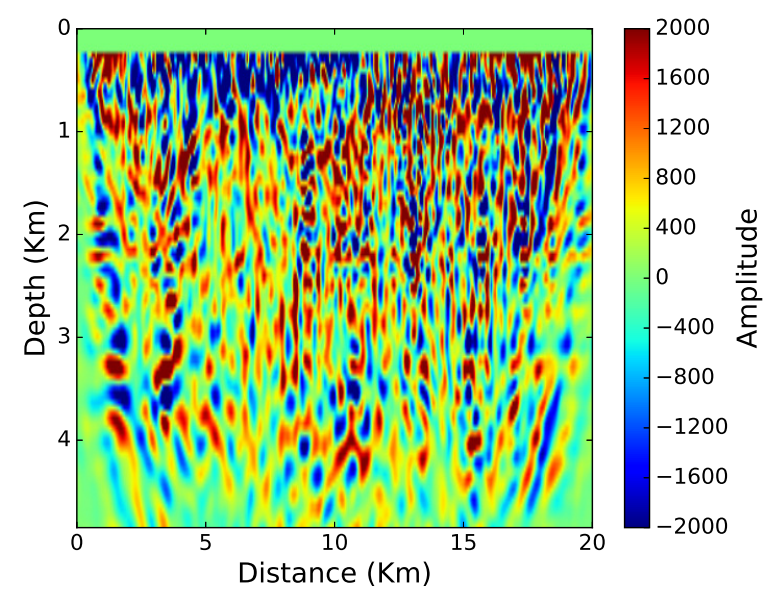

(f)

Figure 1: (a) Gradient Calculation for one shot using a $0^{\circ}$ phase rotation (No rotated). (b) same as (a) but using a $53^{\circ}$ phase rotation. (c) and (d) Gradient for simultaneous shots using polarity encoding and phase rotation encoding. (e) Conventional gradient and (f) Crosstalk noise for phase rotation encoding. 


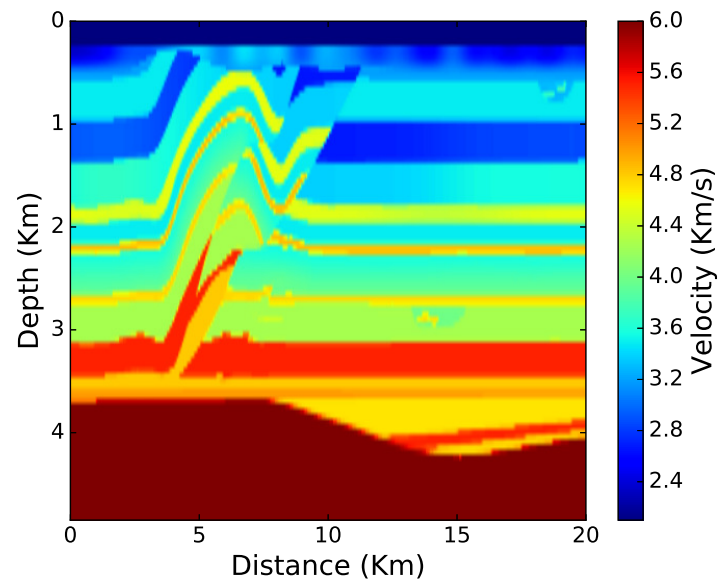

(a)

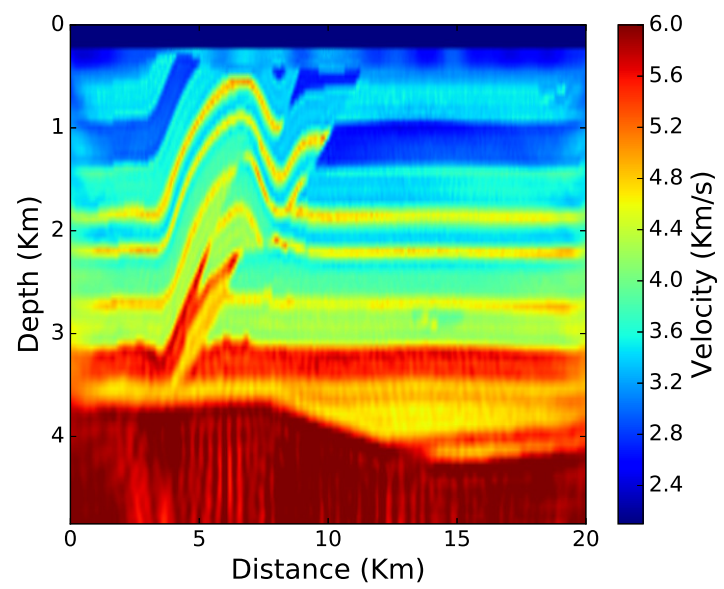

(c)

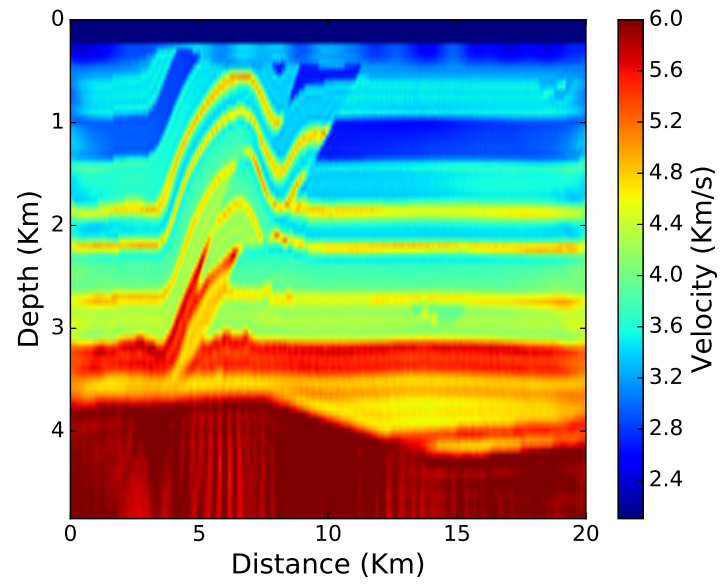

(e)

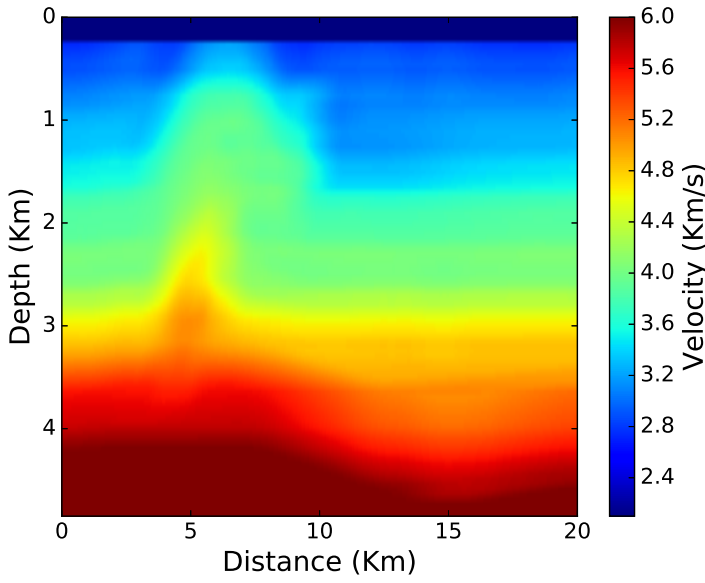

(b)

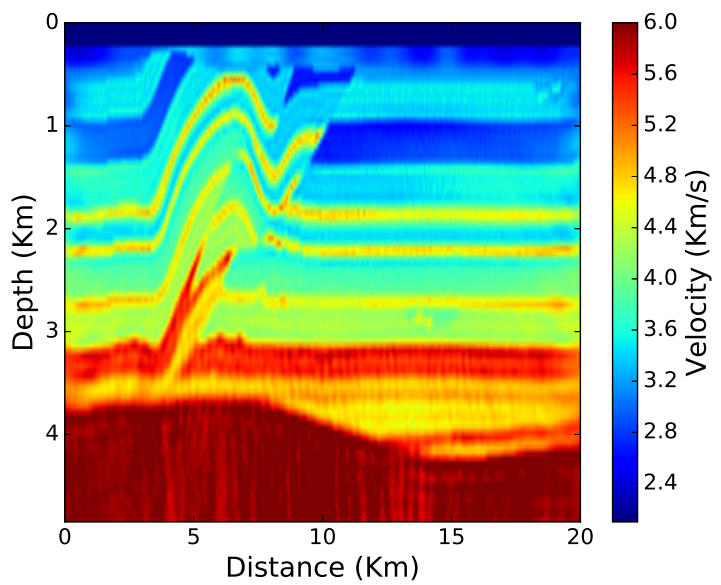

(d)

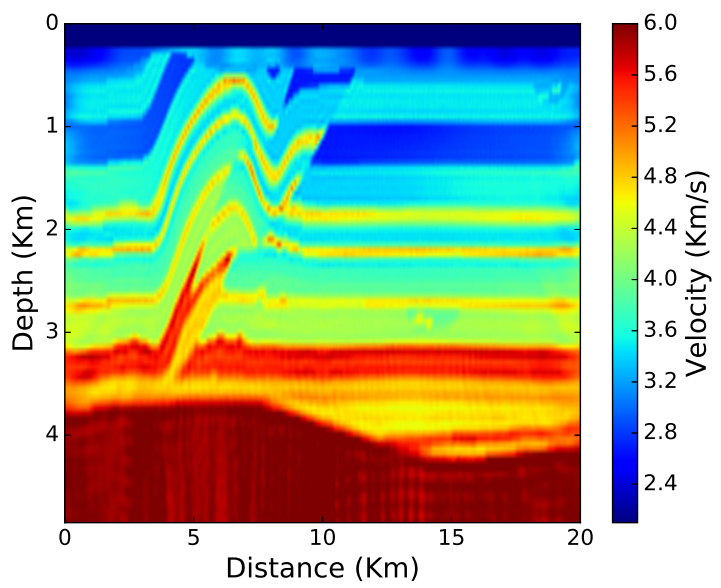

(f)

Figure 2: (a) 2D SEG/EAGE Overthrust Model, (b) Initial model. Partially inverted models after 75 iterations using (c) polarity encoding and (d) phase rotation encoding. Final inverted models after 200 iterations using (e) polarity encoding and (f) phase rotation encoding. 


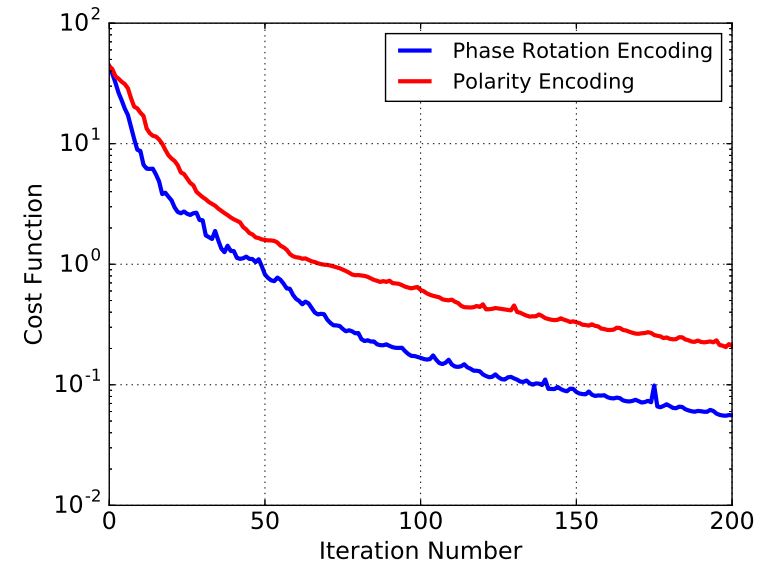

Figure 3: Comparison of Cost Function evolution for polarity and rotation phase encoding strategies.

Encoding multiple shot gathers in prestack migration: 70th SEG meeting, Houston, Expanded Abstracts, 786790.

Komatitsch, D., and R. Martin, 2007, An unsplit convolutional perfectly matched layer improved at grazing incidence for the seismic wave equation: Geophysics, 72, SM155-SM167.

Krebs, J. R., J. E. Anderson, D. Hinkley, R. Neelamani, S. Lee, A. Baumstein, and M.-D. Lacasse, 2009, Fast full-wavefield seismic inversion using encoded sources: Geophysics, 74, WCC177-WCC188.

Lailly, P., 1983, The seismic inversion problem as a sequence of before stack migrations: Conference on
Inverse Scattering, Theory and Application, Society for Industrial and Applied Mathematics, Expanded Abstracts, 206-220.

Nocedal, J., and S. J. Wright, 2006, Numerical optimization, second ed.: Springer.

Pasalic, D., and R. McGarry, 2010, Convolutional perfectly matched layer for isotropic and anisotropic acoustic wave equations: Presented at the SEG Technical Program Expanded Abstracts 2010, Society of Exploration Geophysicists.

Plessix, R.-E., 2006, A review of the adjoint-state method for computing the gradient of a functional with geophysical applications: Geophysical Journal International, 167, 495-503.

Rao, Y., and Y. Wang, 2017, Seismic waveform tomography with shot-encoding using a restarted I-BFGS algorithm: Scientific Reports, 7.

Rao, Y., Y. Wang, and D. Han, 2019, Seismic waveform tomography with simplified restarting scheme: IEEE Geoscience and Remote Sensing Letters, 16, 135-139.

Romero, L. A., D. C. Ghiglia, C. C. Ober, and S. A. Morton, 2000, Phase encoding of shot records in prestack migration: Geophysics, 65, 426-436.

Taner, M. T., F. Koehler, and R. E. Sheriff, 1979, Complex seismic trace analysis: Geophysics, 44, 1041-1063.

Tarantola, A., 1984, Inversion of seismic reflection data in the acoustic approximation: Geophysics, 49, 12591266.

Virieux, J., and S. Operto, 2009, An overview of full-waveform inversion in exploration geophysics: Geophysics, 74, WCC1-WCC26.

Wang, Y., 2007, Seismic time-frequency spectral decomposition by matching pursuit: Geophysics, 72, V13-V20. 